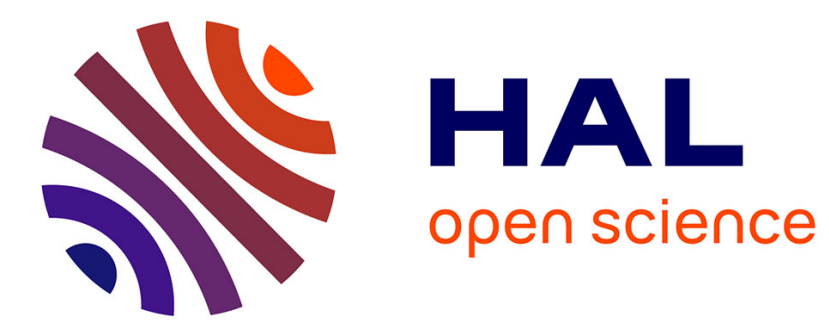

\title{
Properties of a risk measure derived from the expected area in red
}

\author{
Stéphane Loisel, Julien Trufin
}

\section{To cite this version:}

Stéphane Loisel, Julien Trufin. Properties of a risk measure derived from the expected area in red. Insurance: Mathematics and Economics, 2014, 55, pp.191-199. hal-00870224

\section{HAL Id: hal-00870224 \\ https://hal.science/hal-00870224}

Submitted on 6 Oct 2013

HAL is a multi-disciplinary open access archive for the deposit and dissemination of scientific research documents, whether they are published or not. The documents may come from teaching and research institutions in France or abroad, or from public or private research centers.
L'archive ouverte pluridisciplinaire HAL, est destinée au dépôt et à la diffusion de documents scientifiques de niveau recherche, publiés ou non, émanant des établissements d'enseignement et de recherche français ou étrangers, des laboratoires publics ou privés. 


\title{
Properties of a risk measure derived from the expected area in red
}

\author{
STÉPHANE LOISEL ${ }^{\star} \&$ JULIEN TRUFIN ${ }^{\dagger}$ \\ Université de Lyon \\ Université Claude Bernard Lyon 1 \\ Institut de Science Financière et d'Assurances \\ 50 Avenue Tony Garnier, F-69007 Lyon, France \\ E-mail: stephane.loisel@univ-lyon1.fr ${ }^{\star}$ \\ École d'Actuariat \\ Université Laval, Québec, Canada \\ E-mail: julien.trufin@act.ulaval.ca ${ }^{\dagger}$
}




\begin{abstract}
This paper studies a new risk measure derived from the expected area in red introduced in Loisel (2005). Specifically, we derive various properties of a risk measure defined as the smallest initial capital needed to ensure that the expected time-integrated negative part of the risk process on a fixed time interval $[0, T]$ ( $\mathrm{T}$ can be infinite) is less than a given predetermined risk limit. We also investigate the optimal risk limit allocation: given a risk limit set at company level for the sum of the expected areas in red of all lines, we determine the way(s) to allocate this risk limit to the subsequent business lines in order to minimize the overall capital needs.
\end{abstract}

Key words and phrases: Ruin probability, risk measure, expected area in red, stochastic ordering, risk limit. 


\section{Introduction and motivation}

Over the last decade, the concept of risk measures has become very popular in the insurance industry, especially with the introduction of the European Solvency II regulation. Insurance companies are now often required to hold a level of available capital such that the probability for economic ruin after one year is less than 1 in 200. The Value-at-Risk (VaR) risk measure has then emerged as a key instrument in insurance to compute the solvency capital requirement. Readers interested in articles dealing with regulation and solvency for insurance companies could see, e.g., Volume 35, Issue 1 of the Geneva Papers on Risk and Insurance - Issues and Practice. Although this new regulatory framework is an improvement compared to the old insurance practices, it does not consider possible adverse situations in between or beyond the one-year horizon. Ruin theory precisely accounts for the insured risk during the whole life-time of the business or until any given time-horizons. This is why practitioners often look at risks in the ruin context when building internal models. Risk measures derived from ruin theory often provide more robust risk indicators.

Dhaene, Goovaerts and Kaas (2003) give ample motivation for an exponential risk measure inherited from the Cramèr-Lundberg upper bound for the ruin probability in a discretetime ruin model. Cheridito, Delbaen and Kupper (2006) mention (in an application of their study on coherent risk measures for unbounded stochastic processes) a VaR-type risk measure based on the infinite-time ruin probability itself. Overall, over the last few years, the relative position and relation between risk measures that fulfill a list of axioms on the one hand, and classical ruin theory on the other hand, has often been a matter of debate. Trufin, Albrecher and Denuit (2011) take up this issue and investigate in more details some properties of the VaR-type risk measure based on the ultimate ruin probability that is mentioned in Cheridito et al. (2006).

Over the years, the management of the liquidity risk has become a major concern for the insurance industry. The recent financial crisis tells us how this risk can be devastating for a financial institution. In this paper, we propose to study a risk measure derived from ruin theory that takes into account liquidity risk of an insurance business over a given period of time.

In risk theory, many risk measures have been considered for the classical continuous-time risk model. In addition to the well-known finite-time and infinite-time ruin probabilities, some others risk measures have been deeply investigated throughout numerous articles (see Gerber (1988), Dufresne and Gerber (1988) and Picard (1994) for instance). Let us mention the time to ruin, the severity of ruin, the time spent below zero from the first ruin to the first time of recovery, the maximal ruin severity or also the aggregate severity of ruin until recovery. The total time spent below zero has also been studied by dos Reis (1993), using some results of Gerber (1988). However, all those risk measures are either drawn from the infinite-time ruin theory or involve the behavior of the risk process between ruin times and recovery times. This is why Loisel (2005) introduces a risk measure based on a fixed time interval, finite or infinite, i.e. the expected time-integrated negative part of general risk processes on a fixed time interval $[0, T]$, also called the expected area in red. The author derives some expressions for this new risk measure and obtains a closed-form formula in the 
compound Poisson risk model in infinite-time for exponential claims. Biard, Loisel, Macci and Veraverbeke (2010) investigate the asymptotic behavior of the expected area in red and discuss an optimal allocation problem with two business lines.

The expected area in red appears to be a relevant risk indicator for quantifying the liquidity risk of an insurance business over a given time horizon. In this paper, we adopt this risk measure to reflect the liquidity risk of an insurer and we look at the smallest capital that ensures the expected area in red is less than a given level. While the ruin probability focuses on the risk that (at least) one liquidity issue occurs over a finite- or infinite-time horizon, we are here more interested in what happens once the insurer encounters liquidity problems and in controlling their potential impact. Note that in the sequel, the zero surplus does not necessarily correspond to economic ruin. It may correspond to a risk tolerance level, or to a level after which some controls or penalties would affect business and profitability.

The purpose of this paper is to consider certain properties of a risk measure that is derived from the expected area in red. It provides tools to enable a better assessment of the riskiness (through the perspective of the liquidity risk) of certain financial positions in the insurance context. It also makes an interesting link between the initial reserve optimal allocation problem and a new problem of risk budget allocation, that we define and solve. More generally, this paper will contribute to improve the understanding of the connections between the axiomatic framework of risk measures and ruin theory.

Our paper is organized as follows. In Section 2, we set up the scene by presenting the risk process we are dealing with in the present analysis and by recalling the definitions of some stochastic orders used in the following. Next, in Section 3, we introduce the studied risk measure and investigate some of its properties. Further, numerical illustrations are performed within the compound Poisson model for an infinite horizon to illustrate the diversification benefit resulting from the aggregation of two business lines. Finally, Section 4 determines the way(s) to allocate a risk limit set at company level to the subsequent business lines in order to minimize the overall capital needs.

\section{Risk model and stochastic orders}

\subsection{The model}

The surplus process (or risk process) is defined as

$$
U_{t}=u+c t-S_{t}, \quad t \geq 0
$$

where $U_{t}$ is the insurer's capital at time $t$ starting from some initial capital $U_{0}=u, c$ is the (constant) premium income per unit of time and $S_{t}=\sum_{k=1}^{N_{t}} X_{k}$ is the total claim amount up to time $t$, with $N_{t}$ the corresponding number of claims, and $X_{k}$ the size of the $k$ th claim, assumed to be non-negative. The claim number process $\mathbf{N}=\left\{N_{t}, t \geq 0\right\}$ is governed by a sequence of identically distributed inter-occurrence times $T_{k}$ with a common distribution function $F_{T}$. The $X_{k}$ 's are identically distributed as $X$, with distribution function 
$F_{X}$. Let us notice that we do not require the independence of the inter-occurrence times $T_{k}$ 's nor of the claim sizes $X_{k}$ 's. Also, we do not assume the $X_{k}$ 's to be independent from the $T_{k}$ 's.

The expected area in red on a fixed time interval $[0, T]$ is defined as

$$
\mathbb{E}\left[I_{T, c}(u)\right]=\mathbb{E}\left[\int_{t=0}^{T}\left|U_{t}\right| 1_{\left\{U_{t}<0\right\}} d t\right] .
$$

It can also be expressed as follows, which will be useful in our analysis:

$$
\begin{aligned}
\mathbb{E}\left[I_{T, c}(u)\right] & =\mathbb{E}\left[\int_{t=0}^{T}\left|U_{t}\right| 1_{\left\{U_{t}<0\right\}} d t\right] \\
& =\int_{t=0}^{T} \mathbb{E}\left[\left|U_{t}\right| 1_{\left\{U_{t}<0\right\}}\right] d t \quad \text { using Fubini's Theorem } \\
& =\int_{t=0}^{T} \mathbb{E}\left[\left(S_{t}-c t-u\right)_{+}\right] d t .
\end{aligned}
$$

Henceforth, when needed to make explicit the dependence on $\mathbf{S}=\left\{S_{t}, t \geq 0\right\}$, we will denote $\mathbb{E}\left[I_{T, c}(u)\right]$ as $\mathbb{E}\left[I_{T, c}^{(\mathbf{S})}(u)\right]$.

\subsection{Stochastic orders}

In this section, we recall the definitions of some stochastic orders that will be useful in the following. For more details, we refer the interested reader, e.g., to Denuit et al. (2005).

Given two random variables $X$ and $Y, X$ precedes $Y$ in the usual stochastic order, denoted as $X \preceq_{\text {st }} Y$, if

$$
\bar{F}_{X}(u) \leq \bar{F}_{Y}(u) \text { for all } u,
$$

where $\bar{F}_{X}=1-F_{X}$ and $\bar{F}_{Y}=1-F_{Y}$. The later is also equivalent to the inequality $\mathbb{E}[g(X)] \leq \mathbb{E}[g(Y)]$ for any non-decreasing function $g$ such that the expectations exist.

The usual stochastic order compares the sizes of the risks. The convex order focuses on their variabilities. It allows to compare two risks with identical means. Given two random variables $X$ and $Y$ such that $\mathbb{E}[X]=\mathbb{E}[Y], X$ precedes $Y$ in the convex order, denoted as $X \preceq_{\mathrm{cx}} Y$, when

$$
\int_{t}^{\infty} \bar{F}_{X}(u) d u \leq \int_{t}^{\infty} \bar{F}_{Y}(u) d u \text { for all } t .
$$

The inequality in (2.2) can be equivalently written as

$$
\mathbb{E}\left[(X-t)_{+}\right] \leq \mathbb{E}\left[(Y-t)_{+}\right] \quad \text { for all } t .
$$

From (2.3) it follows that $X \preceq_{\mathrm{cx}} Y$ if, and only if, $\mathbb{E}[g(X)] \leq \mathbb{E}[g(Y)]$ for all convex functions $g: \mathbb{R}^{+} \rightarrow \mathbb{R}$, provided the expectations exist. 
The increasing convex, or stop loss, order combines the aspects of size (as $\preceq_{\text {st }}$ ) and variability (as $\preceq_{\mathrm{cx}}$ ). Being smaller in the stop-loss order means "smaller" and "less variable". By definition, $X$ is said to be smaller than $Y$ in the stop-loss order, denoted as $X \preceq_{\text {icx }} Y$, when

$$
\int_{t}^{\infty} \bar{F}_{X}(u) d u \leq \int_{t}^{\infty} \bar{F}_{Y}(u) d u \text { for all } t
$$

or equivalently if

$$
\mathbb{E}\left[(X-t)_{+}\right] \leq \mathbb{E}\left[(Y-t)_{+}\right] \text {for all } t .
$$

Similarly, $X \preceq_{\text {icx }} Y$ if, and only if, $\mathbb{E}[g(X)] \leq \mathbb{E}[g(Y)]$ for all non-decreasing convex functions $g: \mathbb{R}^{+} \rightarrow \mathbb{R}$, provided the expectations exist. Obviously, in case $\mathbb{E}[X]=\mathbb{E}[Y]$, the orders $\preceq_{\text {cx }}$ and $\preceq_{\text {icx }}$ are equivalent.

In the multivariate case, the supermodular order is a useful tool to compare random vectors $\underline{X}=\left(X_{1}, \ldots, X_{n}\right)$ and $\underline{Y}=\left(Y_{1}, \ldots, Y_{n}\right)$ with the same marginals and different levels of dependence. A random vector $X$ is said to be smaller than the random vector $\underline{Y}$ in the supermodular ordering, denoted $\underline{X} \preceq_{\mathrm{sm}} \underline{Y}$, if $\mathbb{E}[f(\underline{X})] \leq \mathbb{E}[f(\underline{Y})]$ for all supermodular functions $f$ such that the expectations exist. A function $f: \mathbb{R}^{n} \rightarrow \mathbb{R}$ is said to be supermodular if

$$
\begin{aligned}
f\left(x_{1}, \ldots, x_{i}+\epsilon, \ldots,\right. & \left.x_{j}+\delta, \ldots, x_{n}\right)-f\left(x_{1}, \ldots, x_{i}+\epsilon, \ldots, x_{j}, \ldots, x_{n}\right) \\
& \geq f\left(x_{1}, \ldots, x_{i}, \ldots, x_{j}+\delta, \ldots, x_{n}\right)-f\left(x_{1}, \ldots, x_{i}, \ldots, x_{j}, \ldots, x_{n}\right)
\end{aligned}
$$

holds for all $\underline{x}=\left(x_{1}, \ldots, x_{n}\right) \in \mathbb{R}^{n}, 1 \leq i<j \leq n$ and all $\epsilon, \delta>0$.

For $n=2$, the supermodular order is equivalent to the positive quadrant dependent order. The random vector $\underline{X}=\left(X_{1}, X_{2}\right)$ is said to be smaller than $\underline{Y}=\left(Y_{1}, Y_{2}\right)$ in the positive quadrant dependent order, denoted $\underline{X} \preceq_{\mathrm{PQD}} \underline{Y}$, if

$$
\operatorname{Pr}\left[X_{1} \leq x_{1}, X_{2} \leq x_{2}\right] \leq \operatorname{Pr}\left[Y_{1} \leq x_{1}, Y_{2} \leq x_{2}\right] \text { for all } x_{1} \text { and } x_{2}
$$

\section{Risk measure derived from the expected area in red}

In Trufin et al. (2011), the authors focus on a risk measure that is the necessary capital such that the ruin probability is less than a given level. In this paper we would like to investigate the capital needed to ensure that the expected area in red is bounded by a predefined value. Namely, for a limit expected area $A>0$, we define

$$
\rho_{A, c}^{(T)}[\mathbf{S}]=\inf \left\{v \geq 0 \mid \mathbb{E}\left[I_{T, c}^{(\mathbf{S})}(v)\right] \leq A\right\}
$$

In words, $\rho_{A, c}^{(T)}[\mathbf{S}]$ is the smallest amount of capital needed such that the expected area in red (on the time interval $[0, T]$ ) for a surplus process with a premium rate $c$ and an aggregate claim amount process $\mathbf{S}$ is at most equal to some specified area $A$.

In what follows, we study the properties of $\rho_{A, c}^{(T)}$ within the axiomatic framework of risk measures. It is worth to mention that unlike in Trufin et al. (2011), we do not restrict our 
analysis to the compound Poisson model nor to an infinite horizon. Moreover, the current risk measure depends this time on the size of the company, which is desirable for concrete applications where liquidity and diversification are at play.

\section{Properties of $\rho_{A, c}^{(T)}$}

Let $\mathbf{S}=\left\{S_{t}, t \geq 0\right\}$ and $\tilde{\mathbf{S}}=\left\{\tilde{S}_{t}, t \geq 0\right\}$ two aggregate claim amount processes with $S_{t}=\sum_{k=1}^{N_{t}} X_{k}$ and $\tilde{S}_{t}=\sum_{k=1}^{\tilde{N}_{t}} \tilde{X}_{k}$. From now on, for a stochastic order relation $\preceq$, the writing $\mathbf{S} \preceq \tilde{\mathbf{S}}$ means $S_{t} \preceq \tilde{S}_{t}$ for all $t \geq 0$. The risk measure $\rho_{A, c}^{(T)}$ possesses the following properties.

Property 3.1. (i) The risk measure $\rho_{A, c}^{(T)}$ agrees with the usual stochastic order, that is $\mathbf{S} \preceq_{s t} \tilde{\mathbf{S}} \Rightarrow \rho_{A, c}^{(T)}[\mathbf{S}] \leq \rho_{A, c}^{(T)}[\tilde{\mathbf{S}}]$.

(ii) The risk measure $\rho_{A, c}^{(T)}$ agrees with the stop-loss order, that is $\mathbf{S} \preceq_{i c x} \tilde{\mathbf{S}} \Rightarrow \rho_{A, c}^{(T)}[\mathbf{S}] \leq$ $\rho_{A, c}^{(T)}[\tilde{\mathbf{S}}]$.

Proof. An equivalent characterization of the usual stochastic order is the existence for all $t$ of random variables $S_{t}^{\prime}$ and $\tilde{S}_{t}^{\prime}$ such that $S_{t}^{\prime}$ (resp. $\tilde{S}_{t}^{\prime}$ ) is distributed as $S_{t}$ (resp. $\left.\tilde{S}_{t}\right)$ and $S_{t}^{\prime} \leq \tilde{S}_{t}^{\prime}$ almost surely (see Theorem 1.A.1 in Shaked and Shanthikumar (2007)). Therefore, we can assume that $S_{t} \leq \tilde{S}_{t}$ for all $t$. This implies $\mathbb{E}\left[\left(S_{t}-c t-u\right)_{+}\right] \leq \mathbb{E}\left[\left(\tilde{S}_{t}-c t-u\right)_{+}\right]$for all $u$ and $t$. Hence by equation (2.1),

$$
\mathbf{S} \preceq_{\text {st }} \tilde{\mathbf{S}} \Rightarrow \mathbb{E}\left[I_{T, c}^{(\mathbf{S})}(u)\right] \leq \mathbb{E}\left[I_{T, c}^{(\tilde{\mathbf{S}})}(u)\right] \quad \text { for all } u .
$$

Turning to item (ii), it suffices to notice that the inequality $\mathbb{E}\left[\left(S_{t}-c t-u\right)_{+}\right] \leq \mathbb{E}\left[\left(\tilde{S}_{t}-c t-u\right)_{+}\right]$ holds true for all $u$ and $t$ (see equation (2.4)). Consequently, by (2.1), after integrating with respect to $t$,

$$
\mathbf{S} \preceq_{\mathrm{icx}} \tilde{\mathbf{S}} \Rightarrow \mathbb{E}\left[I_{T, c}^{(\mathbf{S})}(u)\right] \leq \mathbb{E}\left[I_{T, c}^{(\tilde{\mathbf{S}})}(u)\right] \quad \text { for all } \quad u \text {. }
$$

In particular, if $\mathbf{S} \preceq_{\text {icx }} \tilde{\mathbf{S}}$ and $\mathbb{E}\left[S_{t}\right]=\mathbb{E}\left[\tilde{S}_{t}\right]$ for all $t \geq 0$, i.e. if $\mathbf{S} \preceq_{\text {cx }} \tilde{\mathbf{S}}$, then $\rho_{A, c}^{(T)}[\mathbf{S}] \leq \rho_{A, c}^{(T)}[\tilde{\mathbf{S}}]$.

Remark: As the usual stochastic order implies the stop-loss order, we notice that Property 3.1 (i) can be seen as a particular case of Property 3.1 (ii).

Let us highlight some situations where the stochastic inequality $\mathbf{S} \preceq_{\text {icx }} \tilde{\mathbf{S}}$ holds true.

Example 1: We suppose the $X_{k}$ 's (resp. $\tilde{X}_{k}$ 's) to be independent and identically distributed and to be independent of $\left\{N_{t}, t \geq 0\right\}$ (resp. $\left.\left\{\tilde{N}_{t}, t \geq 0\right\}\right)$.

(a) If $X \preceq_{\text {icx }} \tilde{X}$ and $N_{t} \preceq_{\text {icx }} \tilde{N}_{t}$ for all $t \geq 0$ then $\mathbf{S} \preceq_{\text {icx }} \tilde{\mathbf{S}}$. Indeed, it is well-known that the stop-loss ordering is preserved under compounding of independent risks, see e.g. Property 3.4.39 in Denuit et al. (2005). 
(b) Let $X=V+Y$ and $\tilde{X}=V+Z$ with $V, Y$ and $Z$ three non-negative random variables, $Y$ and $Z$ being identically distributed. If $Z$ is more positively dependent on $V$ than $Y$, in the sense that $(V, Y) \preceq_{\text {sm }}(V, Z)$, and if $N_{t} \preceq_{\text {icx }} \tilde{N}_{t}$ for all $t \geq 0$, then we have $\mathbf{S} \preceq_{\text {icx }} \tilde{\mathbf{S}}$. This is directly deduced from (a) since $V+Y \preceq_{\mathrm{cx}} V+Z$ (see Dhaene and Goovaerts (1996) for instance).

(c) Let $N_{t}=\sum_{i=1}^{m} N_{t}^{i}$ and $\tilde{N}_{t}=\sum_{i=1}^{m} \tilde{N}_{t}^{i}$, where for each $i$, the claim number processes $\left\{N_{t}^{i}, t \geq 0\right\}$ and $\left\{\tilde{N}_{t}^{i}, t \geq 0\right\}$ have the same distribution. For each $t \geq 0$, let $\underline{N}_{t}=$ $\left(N_{t}^{1}, \ldots, N_{t}^{m}\right)$ and $\underline{\tilde{N}}_{t}=\left(\tilde{N}_{t}^{1}, \ldots, \tilde{N}_{t}^{m}\right)$ be two random vectors. If $\underline{N}_{t} \preceq_{\mathrm{sm}} \underline{\tilde{N}}_{t}$ for all $t \geq 0$ and $X \preceq_{\text {icx }} \tilde{\tilde{X}}$, then $\mathbf{S} \preceq_{\text {icx }} \tilde{\mathbf{S}}$. Indeed, from Müller (1997), we have $\underline{N}_{t} \preceq_{\text {sm }} \underline{N}_{t}$ implies $\sum_{i=1}^{m} N_{t}^{i} \preceq_{\mathrm{cx}} \sum_{i=1}^{m} \tilde{N}_{t}^{i}$ for all $t \geq 0$. Then, by (a), we have the announced result.

Remark: If we consider $\mathbf{S}$ and $\tilde{\mathbf{S}}$ such that $\left\{N_{t}, t \geq 0\right\}$ and $\left\{\tilde{N}_{t}, t \geq 0\right\}$ are identically distributed and $\left(X_{1}, \ldots, X_{m}\right) \preceq_{\mathrm{sm}}\left(\tilde{X}_{1}, \ldots, \tilde{X}_{m}\right)$ for all $m$, then, similarly to Example 1 (c), it comes $\mathbf{S} \preceq_{\mathrm{cx}} \tilde{\mathbf{S}}$ since $\sum_{i=1}^{m} X_{i} \preceq_{\mathrm{cx}} \sum_{i=1}^{m} \tilde{X}_{i}$ for all $m$ obviously yields $\sum_{i=1}^{N_{t}} X_{i} \preceq_{\mathrm{cx}} \sum_{i=1}^{N_{t}} \tilde{X}_{i}$.

Example 2: We assume that the bivariate random vectors $\left(T_{k}, X_{k}\right)$ (resp. $\left.\left(\tilde{T}_{k}, \tilde{X}_{k}\right)\right)$ for $k=1,2, \ldots$ are mutually independent. If $T$ and $\tilde{T}$ are identically distributed and if $X$ and $\tilde{X}$ are identically distributed with $(\tilde{X}, \tilde{T}) \preceq_{\mathrm{sm}}(X, T)$, then $\mathbf{S} \preceq_{\mathrm{cx}} \tilde{\mathbf{S}}$. Equivalently, let us prove that $c t-S_{t} \preceq_{\mathrm{cx}} c t-\tilde{S}_{t}$ for all $t \geq 0$. We can write

$$
c t-S_{t}=\sum_{k=1}^{N_{t}}\left(c T_{k}-X_{k}\right)+c\left(t-\sum_{k=1}^{N_{t}} T_{k}\right) .
$$

Therefore, showing that $c t-S_{t} \preceq_{\mathrm{cx}} c t-\tilde{S}_{t}$ for all $t \geq 0$ amounts to demonstrate that $\sum_{k=1}^{n}\left(c T_{k}-X_{k}\right) \preceq_{\mathrm{cx}} \sum_{k=1}^{n}\left(c \tilde{T}_{k}-\tilde{\tilde{X}}_{k}\right)$ for all $n$ (we can assume $N_{s}=\tilde{N}_{s}$ for all $s \leq t$ as the convex order is closed under mixtures). Since the random vectors $\left(T_{k}, X_{k}\right)\left(\operatorname{resp} .\left(\tilde{T}_{k}, \tilde{X}_{k}\right)\right)$ for $k=1,2, \ldots$ are mutually independent on the one hand, and the convex order is closed under convolution on the other hand, it suffices to show that $c T_{k}-X_{k} \preceq$ cx $c \tilde{T}_{k}-\tilde{X}_{k}$ for all k. Now, from Theorem 9.A.18 in Shaked and Shantikhumar $(2007),(\tilde{X}, c \tilde{T}) \preceq_{\text {sm }}(X, c T)$ directly implies $c T-X \preceq$ cx $c \tilde{T}-\tilde{X}$.

We now show that the risk measure $\rho_{A, c}^{(T)}$ also possesses some other properties that are desirable for its application in actuarial science. We note that in the following, by $\mathbf{S}+a$ and $a \mathbf{S}$, we actually mean the claim processes $\left\{S_{t}+a, t \geq 0\right\}$ and $\left\{a S_{t}, t \geq 0\right\}$ respectively.

Property 3.2. (i) The risk measure $\rho_{A, c}^{(T)}$ is monotone, that is $\operatorname{Pr}\left[S_{t} \leq \tilde{S}_{t}\right]=1$ for all $t \geq 0 \Rightarrow$ $\rho_{A, c}^{(T)}[\mathbf{S}] \leq \rho_{A, c}^{(T)}[\tilde{\mathbf{S}}]$.

(ii) The risk measure $\rho_{A, c}^{(T)}$ is translation invariant, that is $\rho_{A, c}^{(T)}[\mathbf{S}+a]=\rho_{A, c}^{(T)}[\mathbf{S}]+$ a for any constant $a>0$.

(iii) The risk measure $\rho_{A, c}^{(T)}$ is positively homogeneous, in the sense that $\rho_{A, c}^{(T)}[a \mathbf{S}]=a \rho_{\frac{A}{a}, \frac{c}{a}}^{(T)}[\mathbf{S}]$ for any constant $a>0$. 
(iv) The risk measure $\rho_{A, c}^{(T)}$ is subadditive, in the sense that the inequality $\rho_{A, c}^{(T)}[\mathbf{S}+\tilde{\mathbf{S}}] \leq$ $\rho_{\gamma A, \alpha c}^{(T)}[\mathbf{S}]+\rho_{(1-\gamma) A,(1-\alpha) c}^{(T)}[\tilde{\mathbf{S}}]$ holds true for all $\alpha, \gamma \in(0,1)$, whatever the dependence structure between the claim processes $\mathbf{S}$ and $\tilde{\mathbf{S}}$.

Proof. The monotonicity immediately follows since by equation (2.1), $\operatorname{Pr}\left[S_{t} \leq \tilde{S}_{t}\right]=1$ for all $t \geq 0$ implies $\mathbb{E}\left[I_{T, c}^{(\mathbf{S})}(u)\right] \leq \mathbb{E}\left[I_{T, c}^{(\tilde{\mathbf{S}})}(u)\right]$ for all $u$. Item (ii) is obvious since adding a deterministic amount to the insurance risk equally increases the necessary initial capital to maintain the same level for the expected area in red. For item (iii), it suffices to prove that $\mathbb{E}\left[I_{T, c}^{(a \mathbf{S})}(u)\right]=a \mathbb{E}\left[I_{T, \frac{c}{a}}^{(\mathbf{S})}\left(\frac{u}{a}\right)\right]$. By equation (2.1), this equality holds true, as we have

$$
\mathbb{E}\left[\left(a S_{t}-c t-u\right)_{+}\right]=a \mathbb{E}\left[\left(S_{t}-\frac{c t}{a}-\frac{u}{a}\right)_{+}\right] .
$$

Considering item (iv), let us first prove that the inequality

$$
\mathbb{E}\left[I_{T, c}^{(\mathbf{S}+\tilde{\mathbf{S}})}(u)\right] \leq \mathbb{E}\left[I_{T, \alpha c}^{(\mathbf{S})}(\beta u)\right]+\mathbb{E}\left[I_{T,(1-\alpha) c}^{(\tilde{\mathbf{S}})}((1-\beta) u)\right]
$$

holds true for all $u$ and $\alpha, \beta \in(0,1)$. By equation (2.1), it suffices to notice that

$$
\begin{aligned}
\mathbb{E}\left[\left(S_{t}+\tilde{S}_{t}-c t-u\right)_{+}\right] & =\mathbb{E}\left[\left(S_{t}-\alpha c t-\beta u+\tilde{S}_{t}-(1-\alpha) c t-(1-\beta) u\right)_{+}\right] \\
& \leq \mathbb{E}\left[\left(S_{t}-\alpha c t-\beta u\right)_{+}\right]+\mathbb{E}\left[\left(\tilde{S}_{t}-(1-\alpha) c t-(1-\beta) u\right)_{+}\right] .
\end{aligned}
$$

Now, from equation (3.1) with $\beta=\frac{\rho_{\gamma A, \alpha c}^{(T)}[\mathbf{S}]}{\rho_{\gamma A, \alpha c}^{(T)}[\mathbf{S}]+\rho_{(1-\gamma) A,(1-\alpha) c}^{(T)}[\tilde{\mathbf{S}}]}$, we have

$$
\begin{aligned}
\mathbb{E}\left[I _ { T , c } ^ { ( \mathbf { S } + \tilde { \mathbf { S } } ) } \left(\rho_{\gamma A, \alpha c}^{(T)}[\mathbf{S}]+\right.\right. & \left.\left.\rho_{(1-\gamma) A,(1-\alpha) c}^{(T)}[\tilde{\mathbf{S}}]\right)\right] \\
& \leq \mathbb{E}\left[I_{T, \alpha c}^{(\mathbf{S})}\left(\rho_{\gamma A, \alpha c}^{(T)}[\mathbf{S}]\right)\right]+\mathbb{E}\left[I_{T,(1-\alpha) c}^{(\tilde{\mathbf{S}})}\left(\rho_{(1-\gamma) A,(1-\alpha) c}^{(T)}[\tilde{\mathbf{S}}]\right)\right] \\
& \left.\leq A \quad \text { (by definition of } \rho_{\gamma A, \alpha c}^{(T)}[\mathbf{S}] \text { and } \rho_{(1-\gamma) A,(1-\alpha) c}^{(T)}[\tilde{\mathbf{S}}]\right) .
\end{aligned}
$$

The notion of positive homogeneity can be interpreted as the independence with respect to the monetary unit used. When considering two different monetary units $u_{1}$ and $u_{2}$ say, with exchange rate $a$ (i.e. with $u_{1}=a u_{2}$ ), the risk limit $A$ set in the first unit becomes logically $A / a$ in the second one, as the premium rate $c$ and the claim process $a \mathbf{S}$ that become respectively $c / a$ and $\mathbf{S}$.

The subadditivity property reflects the idea that the risk can be reduced by diversification. For a fair comparison, we note that the risk limit considered for the aggregate portfolio has been naturally taken as equal to the sum of the risk limits of the subsequent business lines. The diversification benefit resulting from the aggregation of two business lines with premium 
rates $c_{1}=\alpha c$ and $c_{2}=(1-\alpha) c$, claim processes $\mathbf{S}$ and $\tilde{\mathbf{S}}$ and respective risk limits $A_{1}=\gamma A$ and $A_{2}=(1-\gamma) A$ is then positive and is given by

$$
\Delta(\mathbf{S}, \tilde{\mathbf{S}})=\rho_{\gamma A, \alpha c}^{(T)}[\mathbf{S}]+\rho_{(1-\gamma) A,(1-\alpha) c}^{(T)}[\tilde{\mathbf{S}}]-\rho_{A, c}^{(T)}[\mathbf{S}+\tilde{\mathbf{S}}] .
$$

As the risk measure $\rho_{A, c}^{(T)}$ satisfies the four axioms of Property 3.2, one can say that $\rho_{A, c}^{(T)}$ is coherent, bearing in mind the senses brought in our context to the notions of positive homogeneity and subadditivity.

Let us illustrate the diversification benefit for $T=\infty$ in two examples. Beforehand, we recall the following Theorem of Loisel (2005):

Theorem. Within the compound Poisson model, for a positive safety loading $\eta=(c-$ $\left.\lambda \mu_{X}\right) / \lambda \mu_{X}$ (where $\mu_{X}=\mathbb{E}[X]$ ), the expected area in red $\mathbb{E}\left[I_{\infty, c}(u)\right]$ and the ultimate ruin probability $\psi(u)$ are linked by the following equation:

$$
\frac{d^{2}}{d u^{2}} \mathbb{E}\left[I_{\infty, c}(u)\right]=\frac{1}{c} \frac{1}{1-\psi(0)} \psi(u) \quad \text { for all } \quad u>0
$$

In particular, it is possible to get explicit expressions for $\mathbb{E}\left[I_{\infty, c}(u)\right]$ when the claim size distribution is exponential or a combination of exponentials for instance, as we know the ruin probability $\psi(u)$ in these cases.

As an example, for $X \sim \operatorname{Exp}(1 / \mu)$ and a positive safety loading $\eta=(c-\lambda \mu) / \lambda \mu$, we have $\psi(u)=(1-\mu R) e^{-R u}$, where $R=(1 / \mu)(1-\lambda \mu / c)$. Hence, the integration of this well-known formula for $\psi(u)$ directly leads to

$$
\mathbb{E}\left[I_{\infty, c}(u)\right]=\left(\frac{1-\mu R}{c \mu R^{3}}\right) e^{-R u}
$$

since $\mathbb{E}\left[I_{\infty, c}(u)\right]$ tends to 0 as $u \rightarrow \infty$. In this case, our risk measure $\rho_{A, c}^{(\infty)}$ can then be explicitly written as

$$
\rho_{A, c}^{(\infty)}= \begin{cases}\frac{1}{R}\left[\ln \left(\frac{1-\mu R}{c \mu R^{3}}\right)-\ln (A)\right] & \text { for } A<\frac{1-\mu R}{c \mu R^{3}} \\ 0 & \text { otherwise }\end{cases}
$$

Example 3: Let $S_{t}=\sum_{k=1}^{N_{t}} X_{k}$ and $\tilde{S}_{t}=\sum_{k=1}^{\tilde{N}_{t}} \tilde{X}_{k}$, where $\left\{N_{t}, t \geq 0\right\}$ and $\left\{\tilde{N}_{t}, t \geq 0\right\}$ are assumed to be Poisson processes with constant intensities $\lambda$ and $\tilde{\lambda}$ respectively. The $X_{k}$ 's (resp. $\tilde{X}_{k}$ 's) are supposed to be independent and distributed as the generic random variable $X$ (resp. $\tilde{X})$, with $X \sim \operatorname{Exp}(1 / \mu)$ (resp. $\tilde{X} \sim \operatorname{Exp}(1 / \tilde{\mu})$, with $\tilde{\mu} \neq \mu$ ). The claim sizes processes $\left\{X_{k}\right\}$ and $\left\{\tilde{X}_{k}\right\}$ are also assumed to be (1) mutually independent and (2) independent of $\left\{N_{t}, t \geq 0\right\}$ and $\left\{\tilde{N}_{t}, t \geq 0\right\}$. Let us consider $N_{t}$ and $\tilde{N}_{t}$ of the form

$$
N_{t}=N_{t}^{(1)}+N_{t}^{(0)} \text { and } \tilde{N}_{t}=N_{t}^{(2)}+N_{t}^{(0)}
$$

where $\left\{N_{t}^{(0)}, t \geq 0\right\},\left\{N_{t}^{(1)}, t \geq 0\right\}$ and $\left\{N_{t}^{(2)}, t \geq 0\right\}$ are independent Poisson processes with constant rates $\lambda_{0}, \lambda-\lambda_{0}, \tilde{\lambda}-\lambda_{0}$ respectively and $\lambda_{0} \leq \min (\lambda ; \tilde{\lambda})$. The process $\mathbf{S}+\tilde{\mathbf{S}}$ is then also 
compound Poisson with intensity $\lambda+\tilde{\lambda}-\lambda_{0}$ and generic claim size $Y$ with distribution function $F_{Y}=\frac{1}{\lambda+\tilde{\lambda}-\lambda_{0}}\left(\left(\lambda-\lambda_{0}\right) F_{X}+\left(\tilde{\lambda}-\lambda_{0}\right) F_{\tilde{X}}+\lambda_{0} F_{X+\tilde{X}}\right) . \quad$ As $\mu \neq \tilde{\mu}, F_{X+\tilde{X}}=\frac{1}{1-(\tilde{\mu} / \mu)} F_{X}+$ $\frac{1}{1-(\mu / \tilde{\mu})} F_{\tilde{X}}$ and hence $F_{Y}=\alpha_{1} F_{X}+\left(1-\alpha_{1}\right) F_{\tilde{X}}$, where

$$
\alpha_{1}=\frac{1}{\lambda+\tilde{\lambda}-\lambda_{0}}\left(\lambda+\lambda_{0}\left(\frac{1}{1-\tilde{\mu} / \mu}-1\right)\right)
$$

i.e. $F_{Y}$ appears to be a combination of exponential distributions. This well-known special case of claim size distributions is studied in Gerber et al. (1987), and the corresponding ruin probability $\psi(u)$ for a positive safety loading $\eta=\frac{c-\left(\lambda+\tilde{\lambda}-\lambda_{0}\right) \mu_{Y}}{\left(\lambda+\tilde{\lambda}-\lambda_{0}\right) \mu_{Y}}$ is of the form

$$
\psi(u)=C_{1} e^{-r_{1} u}+C_{2} e^{-r_{2} u},
$$

with

$$
C_{j}=\frac{\alpha_{1} /\left(1 / \mu-r_{j}\right)}{\alpha_{1} /\left(1 / \mu-r_{j}\right)^{2}+\alpha_{2} /\left(1 / \tilde{\mu}-r_{j}\right)^{2}} \mu+\frac{\alpha_{2} /\left(1 / \tilde{\mu}-r_{j}\right)}{\alpha_{1} /\left(1 / \mu-r_{j}\right)^{2}+\alpha_{2} /\left(1 / \tilde{\mu}-r_{j}\right)^{2}} \tilde{\mu} \quad j=1,2,
$$

where $\alpha_{2}=1-\alpha_{1}$ and $r_{1}, r_{2}$ are the solutions of the equation

$$
\frac{\lambda+\tilde{\lambda}-\lambda_{0}}{c}\left(\frac{\alpha_{1}}{1 / \mu-r}+\frac{\alpha_{2}}{1 / \tilde{\mu}-r}\right)=1 .
$$

This last equation is equivalent to

$$
r^{2}+r\left(\frac{\lambda+\tilde{\lambda}-\lambda_{0}}{c}-\left(\frac{1}{\mu}+\frac{1}{\tilde{\mu}}\right)\right)+\left[\frac{1}{\mu \tilde{\mu}}-\frac{\lambda+\tilde{\lambda}-\lambda_{0}}{c}\left(\frac{\alpha_{1}}{\tilde{\mu}}+\frac{\alpha_{2}}{\mu}\right)\right]=0 .
$$

We then get

$$
\begin{aligned}
r_{1}=\frac{1}{2}\left(\frac{1}{\mu}\right. & +\frac{1}{\tilde{\mu}}-\frac{\lambda+\tilde{\lambda}-\lambda_{0}}{c} \\
& -\sqrt{\left(\frac{\lambda+\tilde{\lambda}-\lambda_{0}}{c}-\left(\frac{1}{\mu}+\frac{1}{\tilde{\mu}}\right)\right)^{2}-4\left[\frac{1}{\mu \tilde{\mu}}-\frac{\lambda+\tilde{\lambda}-\lambda_{0}}{c}\left(\frac{\alpha_{1}}{\tilde{\mu}}+\frac{\alpha_{2}}{\mu}\right)\right]}
\end{aligned}
$$

and

$$
\begin{aligned}
r_{2}=\frac{1}{2}\left(\frac{1}{\mu}\right. & +\frac{1}{\tilde{\mu}}-\frac{\lambda+\tilde{\lambda}-\lambda_{0}}{c} \\
& +\sqrt{\left.\left(\frac{\lambda+\tilde{\lambda}-\lambda_{0}}{c}-\left(\frac{1}{\mu}+\frac{1}{\tilde{\mu}}\right)\right)^{2}-4\left[\frac{1}{\mu \tilde{\mu}}-\frac{\lambda+\tilde{\lambda}-\lambda_{0}}{c}\left(\frac{\alpha_{1}}{\tilde{\mu}}+\frac{\alpha_{2}}{\mu}\right)\right]\right) .} .
\end{aligned}
$$


So, $\mathbb{E}\left[I_{\infty, c}^{(\mathbf{S}+\tilde{\mathbf{S}})}(u)\right]$ is simply given by

$$
\mathbb{E}\left[I_{\infty, c}^{(\mathbf{S}+\tilde{\mathbf{S}})}(u)\right]=\frac{1}{c} \frac{1}{1-\psi(0)}\left(\frac{C_{1}}{r_{1}^{2}} e^{-r_{1} u}+\frac{C_{2}}{r_{2}^{2}} e^{-r_{2} u}\right)
$$

We assume here that $\lambda=\tilde{\lambda}=1, \mu=2$ and $\tilde{\mu}=3$. Also, we suppose that $\eta=10 \%$ and $A=20$. We then compare $\rho_{\gamma A, c_{1}}^{(\infty)}[\mathbf{S}]+\rho_{(1-\gamma) A, c_{2}}^{(\infty)}[\tilde{\mathbf{S}}]$ and $\rho_{A, c}^{(\infty)}[\mathbf{S}+\tilde{\mathbf{S}}]$ with $c_{1}=\lambda \mu(1+\eta)=2.2$, $c_{2}=\tilde{\lambda} \tilde{\mu}(1+\eta)=3.3$ and $c=c_{1}+c_{2}=5$.5. For $\gamma=\lambda \mu /(\lambda \mu+\tilde{\lambda} \tilde{\mu})=0.4$ (i.e. $\gamma$ is set such that $A$ is allocated to $\mathbf{S}$ and $\tilde{\mathbf{S}}$ in proportion of their expected losses), we get the following results:

\begin{tabular}{|l|c|c|c|c|}
\hline$\lambda_{0}$ & $\rho_{\gamma A, c_{1}}^{(\infty)}[\mathbf{S}]$ & $\rho_{(1-\gamma) A, c_{2}}^{(\infty)}[\tilde{\mathbf{S}}]$ & $\rho_{A, c}^{(\infty)}[\mathbf{S}+\tilde{\mathbf{S}}]$ & $\Delta(\mathbf{S}, \tilde{\mathbf{S}})$ \\
\hline 0 & 123.569 & 185.353 & 123.759 & 185.163 \\
0.1 & 123.569 & 185.353 & 132.049 & 176.874 \\
0.2 & 123.569 & 185.353 & 140.402 & 168.521 \\
0.3 & 123.569 & 185.353 & 148.819 & 160.103 \\
0.4 & 123.569 & 185.353 & 157.300 & 151.623 \\
0.5 & 123.569 & 185.353 & 165.843 & 143.080 \\
0.6 & 123.569 & 185.353 & 174.448 & 134.475 \\
0.7 & 123.569 & 185.353 & 183.113 & 125.809 \\
0.8 & 123.569 & 185.353 & 191.839 & 117.084 \\
0.9 & 123.569 & 185.353 & 200.623 & 108.299 \\
1 & 123.569 & 185.353 & 209.465 & 99.457 \\
\hline
\end{tabular}

As expected, the diversification benefit $\Delta(\mathbf{S}, \tilde{\mathbf{S}})$ is always positive, whatever the value of the dependence parameter $\lambda_{0}$.

For $\lambda_{0} \leq 0.7$, we even observe $\rho_{A, c}^{(\infty)}[\mathbf{S}+\tilde{\mathbf{S}}]$ smaller than $\rho_{(1-\gamma) A, c_{2}}^{(\infty)}[\tilde{\mathbf{S}}]$. This is made possible because the premium rates and areas used to compare these risks, i.e. $\mathbf{S}$ and $\mathbf{S}+\tilde{\mathbf{S}}$, are different, with $c_{2}<c$ and $(1-\gamma) A<A$.

Also, $\Delta(\mathbf{S}, \tilde{\mathbf{S}})$ decreases with the dependence parameter $\lambda_{0}$, which was expected from Example $1(c)$ as for $\epsilon_{1}<\epsilon_{2}$, we have $\left.\left.\left(N_{t}, \tilde{N}_{t}\right)\right|_{\lambda_{0}=\epsilon_{1}} \preceq_{\text {sm }}\left(N_{t}, \tilde{N}_{t}\right)\right|_{\lambda_{0}=\epsilon_{2}}$.

In the case where $\lambda_{0}=1$, i.e. when $N_{t}=\tilde{N}_{t}$, we still observe a diversification benefit. This was expected as for each $k$, the claim sizes $X_{k}$ and $\tilde{X}_{k}$ have been assumed to be independent. From Example $1(b), X_{k}$ and $\tilde{X}_{k}$ positively dependent will lead to a larger $\left.\rho_{A, c}^{(\infty)}[\mathbf{S}+\tilde{\mathbf{S}}]\right|_{\lambda_{0}=1}$. If we consider the extreme case where $X_{k}$ and $\tilde{X}_{k}$ are comonotonic, we simply get $X+\tilde{X} \sim \operatorname{Exp}(1 /(\mu+\tilde{\mu}))$, which leads to $\left.\rho_{A, c}^{(\infty)}[\mathbf{S}+\tilde{\mathbf{S}}]\right|_{\lambda_{0}=1}=308.922$ and hence to $\Delta(\mathbf{S}, \tilde{\mathbf{S}})=0$.

Example 4: Let us consider the two dependent claim processes $S_{t}=\sum_{k=1}^{N_{t}} X_{k}$ and $\tilde{S}_{t}=$ $\sum_{k=1}^{\tilde{N}_{t}} \tilde{X}_{k}$ of Example 3. The claim processes are linked by some events which create claims in both claim streams (common shocks). In this example, we also suppose that the claim 
sizes $X_{k} \mathrm{~s}$ and $\tilde{X}_{k} \mathrm{~s}$ are conditionally independent with respect to a mixing random variable $\Theta$, with

$$
\operatorname{Pr}\left[X_{1}>x_{1}, \ldots, X_{n}>x_{n}, \tilde{X}_{1}>\tilde{x}_{1}, \ldots, \tilde{X}_{p}>\tilde{x}_{p} \mid \Theta=\theta\right]=e^{-\frac{\theta}{\mu}\left(x_{1}+\cdots+x_{n}\right)-\frac{\theta}{\tilde{\mu}}\left(\tilde{x}_{1}+\cdots+\tilde{x}_{p}\right)} .
$$

The unconditioned distribution of each $X_{k}$ and $\tilde{X}_{k}$ is then completely monotone and the dependence structure between the claim sizes (common mixing) is described by a survival Archimedean copula whose generator is the inverse Laplace transform of $\Theta$ (see Albrecher et al. (2011) for more details). Obviously, when $\Theta=\theta$, we are back in the model of Example 3 .

Let us assume that the safety loading condition is always fulfilled, i.e. $\operatorname{Pr}\left[\Theta \geq \theta_{0}\right]=1$, with $\theta_{0}=\max \left(\frac{\lambda \mu}{c_{1}} ; \frac{\tilde{\lambda} \tilde{\mu}}{c_{2}}\right)$. Hence, by (3.5), the conditional expected area in red $\mathbb{E}\left[I_{\infty, c}^{(\mathbf{S}+\tilde{\mathbf{S}})}(u) \mid \Theta=\right.$ $\theta$ ] can be written as

$$
\mathbb{E}\left[I_{\infty, c}^{(\mathbf{S}+\tilde{\mathbf{S}})}(u) \mid \Theta=\theta\right]=\frac{1}{c} \frac{1}{1-\psi_{\theta}(0)}\left(\frac{C_{1}(\theta)}{\left[r_{1}(\theta)\right]^{2}} e^{-r_{1}(\theta) u}+\frac{C_{2}(\theta)}{\left[r_{2}(\theta)\right]^{2}} e^{-r_{2}(\theta) u}\right)
$$

with

$$
\psi_{\theta}(0)=C_{1}(\theta)+C_{2}(\theta),
$$

where $C_{1}(\theta)$ and $C_{2}(\theta)$ (resp. $r_{1}(\theta)$ and $r_{2}(\theta)$ ) are given by equation (3.2) (resp. equations (3.3) and (3.4)), replacing $\mu$ and $\tilde{\mu}$ with $\mu / \theta$ and $\tilde{\mu} / \theta$ respectively. The unconditioned expected area in red $\mathbb{E}\left[I_{\infty, c}^{(\mathbf{S}+\tilde{\mathbf{S}})}(u)\right]$ is thus of the form

$$
\mathbb{E}\left[I_{\infty, c}^{(\mathbf{S}+\tilde{\mathbf{S}})}(u)\right]=\frac{1}{c} \int_{\theta=\theta_{0}}^{+\infty} \frac{1}{1-\psi_{\theta}(0)}\left(\frac{C_{1}(\theta)}{\left[r_{1}(\theta)\right]^{2}} e^{-r_{1}(\theta) u}+\frac{C_{2}(\theta)}{\left[r_{2}(\theta)\right]^{2}} e^{-r_{2}(\theta) u}\right) d F_{\Theta}(\theta),
$$

where $F_{\Theta}$ is the distribution function of $\Theta$.

Similarly, $\mathbb{E}\left[I_{\infty, c_{1}}^{(\mathbf{S})}(u)\right]$ and $\mathbb{E}\left[I_{\infty, c_{2}}^{(\tilde{\mathbf{S}})}(u)\right]$ can be expressed as

$$
\mathbb{E}\left[I_{\infty, c_{1}}^{(\mathbf{S})}(u)\right]=\int_{\theta=\frac{\lambda \mu}{c_{1}}}^{+\infty}\left(\frac{1-\mu R_{1}(\theta) / \theta}{c_{1} \mu\left[R_{1}(\theta)\right]^{3} / \theta}\right) e^{-R_{1}(\theta) u} d F_{\Theta}(\theta)
$$

and

$$
\mathbb{E}\left[I_{\infty, c_{2}}^{(\tilde{\mathbf{S}})}(u)\right]=\int_{\theta=\frac{\tilde{\lambda} \tilde{\mu}}{c_{2}}}^{+\infty}\left(\frac{1-\tilde{\mu} R_{2}(\theta) / \theta}{c_{2} \tilde{\mu}\left[R_{2}(\theta)\right]^{3} / \theta}\right) e^{-R_{2}(\theta) u} d F_{\Theta}(\theta)
$$

with

$$
R_{1}(\theta)=\frac{\theta}{\mu}\left(1-\frac{\lambda \mu}{c_{1} \theta}\right) \quad \text { and } \quad R_{2}(\theta)=\frac{\theta}{\tilde{\mu}}\left(1-\frac{\tilde{\lambda} \tilde{\mu}}{c_{2} \theta}\right) .
$$

Let us assume that $\Theta \sim U n i(a, b)$. It comes $\mathbb{E}[X]=\mu \mathbb{E}[1 / \Theta]=\frac{\mu}{b-a} \ln \left(\frac{b}{a}\right)$ and $\mathbb{E}[\tilde{X}]=$ $\tilde{\mu} \mathbb{E}[1 / \Theta]=\frac{\tilde{\mu}}{b-a} \ln \left(\frac{b}{a}\right)$. We also suppose that $\mathbb{E}[X]=2, \mathbb{E}[\tilde{X}]=3, \lambda=\tilde{\lambda}=1, \eta=35 \%$ and $A=20$. Again, we aim to compare $\rho_{\gamma A, c_{1}}^{(\infty)}[\mathbf{S}]+\rho_{(1-\gamma) A, c_{2}}^{(\infty)}[\tilde{\mathbf{S}}]$ and $\rho_{A, c}^{(\infty)}[\mathbf{S}+\tilde{\mathbf{S}}]$ with $c_{1}=\lambda \mathbb{E}[X](1+$ $\eta)=2.7, c_{2}=\tilde{\lambda} \mathbb{E}[\tilde{X}](1+\eta)=4.05, c=c_{1}+c_{2}=6.75$ and $\gamma=\lambda \mathbb{E}[X] /(\lambda \mathbb{E}[X]+\tilde{\lambda} \mathbb{E}[\tilde{X}])=0.4$. We note that the requirement $\operatorname{Pr}\left[\Theta \geq \theta_{0}\right]=1$ becomes $\frac{b}{a} \leq 1+\ln \left(\frac{b}{a}\right)(1+\eta)$ in this case, so that for $b=10, a \geq 5.639$. The next tables summarize the results we obtain: 


\begin{tabular}{|l|c|c|c|c|c|}
\hline \multicolumn{5}{|c|}{$\mathrm{a}=9, \mathrm{~b}=10$} \\
\hline$\lambda_{0}$ & $\rho_{\gamma A, c_{1}}^{(\infty)}[\mathbf{S}]$ & $\rho_{(1-\gamma) A, c_{2}}^{(\infty)}[\tilde{\mathbf{S}}]$ & $\rho_{A, c}^{(\infty)}[\mathbf{S}+\tilde{\mathbf{S}}]$ & $\Delta(\mathbf{S}, \tilde{\mathbf{S}})$ & $\frac{\Delta(\mathbf{S}, \tilde{\mathbf{S}})}{\rho_{\gamma A, c_{1}}^{(\infty)}[\mathbf{S}]+\rho_{(1-\gamma) A, c_{2}}^{(\infty)}[\tilde{\mathbf{S}}]}$ \\
\hline 0 & 17.374 & 26.061 & 8.869 & 34.565 & $79.580 \%$ \\
0.2 & 17.374 & 26.061 & 11.624 & 31.810 & $73.237 \%$ \\
0.4 & 17.374 & 26.061 & 14.459 & 28.975 & $66.710 \%$ \\
0.6 & 17.374 & 26.061 & 17.367 & 26.065 & $60.012 \%$ \\
0.8 & 17.374 & 26.061 & 20.345 & 23.091 & $53.162 \%$ \\
1 & 17.374 & 26.061 & 23.388 & 20.046 & $46.153 \%$ \\
\hline
\end{tabular}

\begin{tabular}{|l|c|c|c|c|c|}
\hline \multicolumn{5}{|c|}{$\mathrm{a}=8, \mathrm{~b}=10$} \\
\hline$\lambda_{0}$ & $\rho_{\gamma A, c_{1}}^{(\infty)}[\mathbf{S}]$ & $\rho_{(1-\gamma) A, c_{2}}^{(\infty)}[\tilde{\mathbf{S}}]$ & $\rho_{A, c}^{(\infty)}[\mathbf{S}+\tilde{\mathbf{S}}]$ & $\Delta(\mathbf{S}, \tilde{\mathbf{S}})$ & $\frac{\Delta(\mathbf{S}, \tilde{\mathbf{S}})}{\rho_{\gamma A, c_{1}}^{(\infty)}[\mathbf{S}]+\rho_{(1-\gamma) A, c_{2}}^{(\infty)}[\tilde{\mathbf{S}}]}$ \\
\hline 0 & 23.972 & 35.958 & 13.977 & 45.954 & $76.678 \%$ \\
0.2 & 23.972 & 35.958 & 17.640 & 42.290 & $70.566 \%$ \\
0.4 & 23.972 & 35.958 & 21.427 & 38.503 & $64.247 \%$ \\
0.6 & 23.972 & 35.958 & 25.330 & 34.600 & $57.734 \%$ \\
0.8 & 23.972 & 35.958 & 29.344 & 30.586 & $51.036 \%$ \\
1 & 23.972 & 35.958 & 33.465 & 26.466 & $44.161 \%$ \\
\hline
\end{tabular}

\begin{tabular}{|l|c|c|c|c|c|}
\hline \multicolumn{5}{|c|}{$\mathrm{a}=7, \mathrm{~b}=10$} \\
\hline$\lambda_{0}$ & $\rho_{\gamma A, c_{1}}^{(\infty)}[\mathbf{S}]$ & $\rho_{(1-\gamma) A, c_{2}}^{(\infty)}[\tilde{\mathbf{S}}]$ & $\rho_{A, c}^{(\infty)}[\mathbf{S}+\tilde{\mathbf{S}}]$ & $\Delta(\mathbf{S}, \tilde{\mathbf{S}})$ & $\frac{\Delta(\mathbf{S}, \tilde{\mathbf{S}})}{\rho_{\gamma A, c_{1}}^{(\infty)}[\mathbf{S}] \rho_{(1-\gamma) A, c_{2}}^{(\infty)}[\tilde{\mathbf{S}}]}$ \\
\hline 0 & 49.462 & 74.192 & 35.307 & 88.346 & $71.446 \%$ \\
0.2 & 49.462 & 74.192 & 42.572 & 81.081 & $65.571 \%$ \\
0.4 & 49.462 & 74.192 & 50.097 & 73.557 & $59.486 \%$ \\
0.6 & 49.462 & 74.192 & 57.865 & 65.788 & $53.204 \%$ \\
0.8 & 49.462 & 74.192 & 65.865 & 57.789 & $46.734 \%$ \\
1 & 49.462 & 74.192 & 74.083 & 49.570 & $40.088 \%$ \\
\hline
\end{tabular}

\begin{tabular}{|l|c|c|c|c|c|}
\hline \multicolumn{6}{|c|}{$\mathrm{a}=6, \mathrm{~b}=10$} \\
\hline$\lambda_{0}$ & $\rho_{\gamma A, c_{1}}^{(\infty)}[\mathbf{S}]$ & $\rho_{(1-\gamma) A, c_{2}}^{(\infty)}[\tilde{\mathbf{S}}]$ & $\rho_{A, c}^{(\infty)}[\mathbf{S}+\tilde{\mathbf{S}}]$ & $\Delta(\mathbf{S}, \tilde{\mathbf{S}})$ & $\frac{\Delta(\mathbf{S}, \tilde{\mathbf{S}})}{\rho_{\gamma A, c_{1}}^{(\infty)}[\mathbf{S}]+\rho_{(1-\gamma) A, c_{2}}^{(\infty)}[\tilde{\mathbf{S}}]}$ \\
\hline 0 & 322.672 & 484.009 & 306.266 & 500.415 & $62.033 \%$ \\
0.2 & 322.672 & 484.009 & 350.758 & 455.922 & $56.518 \%$ \\
0.4 & 322.672 & 484.009 & 396.450 & 410.231 & $50.854 \%$ \\
0.6 & 322.672 & 484.009 & 443.265 & 363.414 & $45.051 \%$ \\
0.8 & 322.672 & 484.009 & 491.137 & 315.544 & $39.116 \%$ \\
1 & 322.672 & 484.009 & 540.003 & 266.677 & $33.059 \%$ \\
\hline
\end{tabular}


In particular, we note that for a given $\lambda_{0}$, a lower $a$ leads to a lower relative diversification benefit $\Delta(\mathbf{S}, \tilde{\mathbf{S}}) /\left(\rho_{\gamma A, c_{1}}^{(\infty)}[\mathbf{S}]+\rho_{(1-\gamma) A, c_{2}}^{(\infty)}[\tilde{\mathbf{S}}]\right)$, which is in line with the expectations since a lower $a$ implies a stronger dependency between $\mathbf{S}$ and $\tilde{\mathbf{S}}$.

\section{Optimal allocation}

Most insurance companies have several business lines (e.g. motor insurance, fire insurance, ...). Decision makers may choose a risk limit at the company level (for the aggregate surplus) if all results are fully consolidated and if they do not want to have a view by line of business. In this case, the risk limit would be the maximal expected area in red that the company is ready to accept for its aggregate surplus. But more and more insurance companies define risk limits not only at company level, but also at the level of business lines. This means that the company could set a risk limit $A$ that would be the maximal acceptable sum of expected areas in red. Henceforth, we denote by $c_{i}$ and $\mathbf{S}_{i}$ the premium rate and the claim process associated to line of business $i(i=1,2, \ldots, K)$. For the sake of clarity, let us first consider a company with two business lines $(K=2)$. The following optimal allocation immediately arises: how to split this global risk limit $A$ into risk limits by line of business $A_{1}$ and $A_{2}$ satisfying $A_{1}+A_{2}=A$ in order to minimize the global required initial surplus

$$
\rho_{A_{1}, c_{1}}^{(T)}\left[\mathbf{S}_{1}\right]+\rho_{A_{2}, c_{2}}^{(T)}\left[\mathbf{S}_{2}\right] .
$$

Note that for $u_{1}, u_{2} \geq 0$,

$$
0 \leq \mathbb{E}\left[I_{T, c_{1}}^{\left(\mathbf{S}_{1}\right)}\left(u_{1}\right)\right]+\mathbb{E}\left[I_{T, c_{2}}^{\left(\mathbf{S}_{2}\right)}\left(u_{2}\right)\right] \leq \mathbb{E}\left[I_{T, c_{1}}^{\left(\mathbf{S}_{1}\right)}(0)\right]+\mathbb{E}\left[I_{T, c_{2}}^{\left(\mathbf{S}_{2}\right)}(0)\right] .
$$

Consequently, for

$$
A \geq \mathbb{E}\left[I_{T, c_{1}}^{\left(\mathbf{S}_{1}\right)}(0)\right]+\mathbb{E}\left[I_{T, c_{2}}^{\left(\mathbf{S}_{2}\right)}(0)\right],
$$

optimal allocations are trivial and correspond to non-negative couples $\left(A_{1}^{*}, A_{2}^{*}\right)$ satisfying

$$
A_{1}^{*} \geq \mathbb{E}\left[I_{T, c_{1}}^{\left(\mathbf{S}_{1}\right)}(0)\right] \text { and } A_{2}^{*} \geq \mathbb{E}\left[I_{T, c_{2}}^{\left(\mathbf{S}_{2}\right)}(0)\right] .
$$

In that case the required initial surplus is zero for both lines:

$$
\rho_{A_{1}^{*}, c_{1}}^{(T)}\left[\mathbf{S}_{1}\right]+\rho_{A_{2}^{*}, c_{2}}^{(T)}\left[\mathbf{S}_{2}\right]=0 .
$$

In the interesting case where $A<\mathbb{E}\left[I_{T, c_{1}}^{\left(\mathbf{S}_{1}\right)}(0)\right]+\mathbb{E}\left[I_{T, c_{2}}^{\left(\mathbf{S}_{2}\right)}(0)\right]$, we now show that the optimal risk limit allocation leads to solutions that are similar to the ones of the optimal initial reserve allocation problem considered in Loisel (2005).

Proposition 4.1. Consider an insurance company with two business lines as described above. Assume that $A<\mathbb{E}\left[I_{T, c_{1}}^{\left(\mathbf{S}_{1}\right)}(0)\right]+\mathbb{E}\left[I_{T, c_{2}}^{\left(\mathbf{S}_{2}\right)}(0)\right]$. The optimal risk limit allocation $\left(A_{1}^{*}, A_{2}^{*}\right)$ either satisfies

$$
\mathbb{E}\left[\tau_{1}\left(A_{1}^{*}\right)\right]=\mathbb{E}\left[\tau_{2}\left(A_{2}^{*}\right)\right]
$$

or is degenerated, i.e. either

$$
A_{1}^{*}=\mathbb{E}\left[I_{T, c_{1}}^{\left(\mathbf{S}_{1}\right)}(0)\right] \text { or } A_{2}^{*}=\mathbb{E}\left[I_{T, c_{2}}^{\left(\mathbf{S}_{2}\right)}(0)\right] .
$$


Note that degenerated cases correspond to extremely unbalanced cases, where one line of business is much more risky than the other one, and needs all the initial surplus. For example, if line of business 1 is too risky in comparison to line of business 2 , then we have

$$
A_{2}^{*}=\mathbb{E}\left[I_{T, c_{2}}^{\left(\mathbf{S}_{2}\right)}(0)\right] \text { and } A_{1}^{*}=A-\mathbb{E}\left[I_{T, c_{2}}^{\left(\mathbf{S}_{2}\right)}(0)\right]<\mathbb{E}\left[I_{T, c_{1}}^{\left(\mathbf{S}_{1}\right)}(0)\right]
$$

In this degenerated case, line 2 starts with zero surplus. One gives the maximal risk limit to line 2 that corresponds to the weakest constraint. More pressure is placed over line 1 .

Proof. For any line of business, $\mathbb{E}\left[I_{\infty, c}^{(\mathbf{S})}(u)\right]$ is strictly convex (see Loisel (2005)) and increases with $u$. Therefore, $h(A)=\rho_{A, c}^{(T)}[\mathbf{S}]$ is also strictly convex and increases with $A$. As we have

$$
\frac{d}{d u} \mathbb{E}\left[I_{\infty, c}^{(\mathbf{S})}(u)\right]=-\mathbb{E}[\tau(u)]
$$

we can deduce that

$$
\frac{\partial}{\partial A} \rho_{A, c}^{(T)}[\mathbf{S}]=-\frac{1}{\mathbb{E}\left[\tau_{A}\right]}
$$

We now denote $A_{1}=\gamma A$, with $0 \leq \gamma \leq 1$, and $A_{2}=(1-\gamma) A$. For line of business 1 , we have

$$
\frac{\partial}{\partial \gamma} \rho_{\gamma A, c_{1}}^{(T)}\left[\mathbf{S}_{1}\right]=\left.\frac{\partial}{\partial A} \rho_{A, c_{1}}^{(T)}\left[\mathbf{S}_{1}\right]\right|_{A=\gamma A} \frac{\partial}{\partial \gamma} \gamma A=-\frac{1}{\mathbb{E}\left[\tau_{1}(\gamma A)\right]} A .
$$

For line of business 2, we have

$$
\frac{\partial}{\partial \gamma} \rho_{(1-\gamma) A, c_{2}}^{(T)}\left[\mathbf{S}_{2}\right]=-\frac{1}{\mathbb{E}\left[\tau_{2}((1-\gamma) A)\right]} \frac{\partial}{\partial \gamma}(1-\gamma) A=\frac{1}{\mathbb{E}\left[\tau_{2}((1-\gamma) A)\right]} A .
$$

Consequently, if the optimal value $\gamma^{*}$ is in the interior of the interval $[0,1]$, we must have

$$
\left.\frac{\partial}{\partial \gamma}\left\{\rho_{\gamma A, c_{1}}^{(T)}\left[\mathbf{S}_{1}\right]+\rho_{(1-\gamma) A, c_{2}}^{(T)}\left[\mathbf{S}_{2}\right]\right\}\right|_{\gamma=\gamma^{*}}=-\frac{1}{\mathbb{E}\left[\tau_{1}\left(\gamma^{*} A\right)\right]} A+\frac{1}{\mathbb{E}\left[\tau_{2}\left(\left(1-\gamma^{*}\right) A\right)\right]} A=0,
$$

which is equivalent to

$$
\mathbb{E}\left[\tau_{1}\left(\gamma^{*} A\right)\right]=\mathbb{E}\left[\tau_{2}\left(\left(1-\gamma^{*}\right) A\right)\right]
$$

Example: We consider the same assumptions as in Example 3. Let $A<\min \left(\frac{1-\mu_{1} R_{1}}{c_{1} \mu_{1} R_{1}^{3}} ; \frac{1-\mu_{2} R_{2}}{c_{2} \mu_{2} R_{2}^{3}}\right)$.

We then have

$$
\rho_{\gamma A, c_{1}}^{(\infty)}\left[\mathbf{S}_{1}\right]=\frac{1}{R_{1}}\left[\ln \left(\frac{1-\mu_{1} R_{1}}{c_{1} \mu_{1} R_{1}^{3}}\right)-\ln (\gamma A)\right]
$$

and

$$
\rho_{(1-\gamma) A, c_{2}}^{(\infty)}\left[\mathbf{S}_{2}\right]=\frac{1}{R_{2}}\left[\ln \left(\frac{1-\mu_{2} R_{2}}{c_{2} \mu_{2} R_{2}^{3}}\right)-\ln ((1-\gamma) A)\right]
$$

Hence, we get

$$
\frac{\partial}{\partial \gamma} \rho_{\gamma A, c_{1}}^{(\infty)}\left[\mathbf{S}_{1}\right]=-\frac{1}{R_{1}} \frac{\partial}{\partial \gamma} \ln (\gamma)=-\frac{1}{\gamma R_{1}}
$$


and

$$
\frac{\partial}{\partial \gamma} \rho_{(1-\gamma) A, c_{2}}^{(\infty)}\left[\mathbf{S}_{2}\right]=-\frac{1}{R_{2}} \frac{\partial}{\partial \gamma} \ln (1-\gamma)=\frac{1}{(1-\gamma) R_{2}}
$$

Therefore, we find that

$$
\left.\frac{\partial}{\partial \gamma}\left\{\rho_{\gamma A, c_{1}}^{(\infty)}\left[\mathbf{S}_{1}\right]+\rho_{(1-\gamma) A, c_{2}}^{(\infty)}\left[\mathbf{S}_{2}\right]\right\}\right|_{\gamma=\gamma^{*}}=-\frac{1}{\gamma^{*} R_{1}}+\frac{1}{\left(1-\gamma^{*}\right) R_{2}}=0
$$

if and only if

$$
\frac{\gamma^{*}}{1-\gamma^{*}}=\frac{R_{2}}{R_{1}}
$$

This is well equivalent to $\mathbb{E}\left[\tau_{1}\left(\gamma^{*} A\right)\right]=\mathbb{E}\left[\tau_{2}\left(\left(1-\gamma^{*}\right) A\right)\right]$ since we have

$$
\mathbb{E}\left[\tau_{1}(\gamma A)\right]=\gamma A R_{1} \quad \text { and } \quad \mathbb{E}\left[\tau_{2}((1-\gamma) A)\right]=(1-\gamma) A R_{2}
$$

In conclusion we are now ready to state the result that generalizes Proposition 4.1 for $K \geq 2$ business lines.

Proposition 4.2. Consider an insurance company with $K \geq 2$ business lines. Assume that $A<\mathbb{E}\left[I_{T, c_{1}}^{\left(\mathbf{S}_{1}\right)}(0)\right]+\cdots+\mathbb{E}\left[I_{T, c_{K}}^{\left(\mathbf{S}_{K}\right)}(0)\right]$. The optimal risk limit allocation $\left(A_{1}^{*}, \ldots, A_{K}^{*}\right)$ can be described as follows: there exists a subset $\mathcal{J} \subset\{1,2, \ldots, K\}$ such that for $j \in \mathcal{J}$, $A_{j}^{*}=\mathbb{E}\left[I_{T, c_{j}}^{\left(\mathbf{S}_{j}\right)}(0)\right]$ and for $k, l \in\{1,2, \ldots, K\} \backslash \mathcal{J}$,

$$
\mathbb{E}\left[\tau_{k}\left(A_{k}^{*}\right)\right]=\mathbb{E}\left[\tau_{l}\left(A_{l}^{*}\right)\right]
$$

Proof. The proof is similar to the one of Proposition 4.1.

Note that $\mathcal{J}$ corresponds to the set of numbers of business lines that are much less risky than the other ones and start with zero initial surplus. If $\mathcal{J}$ is empty, then there is no saturation, and the optimal risk limit allocation is obtained by making the time spent in the red by each line equal to one another.

\section{Conclusion}

In this paper we proposed a new risk measure and showed that it fulfills interesting properties. On contrary to the risk measure discussed in Trufin et al. (2011), the size of the portfolio considered influences $\rho_{A, c}^{(T)}$, which is of practical relevance when dealing with a risk measure that aims to represent a capital. Moreover the present study has been conducted within a more general risk model than the classical compound Poisson model. We also quantified in two numerical examples the diversification benefit obtained by an insurer composed of two business lines. Finally, we showed that the optimal risk budget allocation problem that arises in our context is dual to the optimal initial reserve allocation problem.

Most results might be generalized to other penalty functions. For instance, we could penalize more very negative values than negative values close to zero, as suggested in Picard 
(1994) in his study of the cost of recovery. So, one might extend most results of the present paper to quantities

$$
\mathbb{E}\left[\int_{0}^{T} g\left(\left|U_{t}\right|\right) 1_{\left\{U_{t}<0\right\}} d t\right]
$$

mentioned in Loisel (2005), with $g$ an increasing convex function (to be consistent with the theory of utility functions) such that $g(0)=0$.

\section{Acknowledgements}

Stéphane Loisel thanks the financial support of Milliman under the research chair Actuariat Durable and BNP Paribas Cardif under the research chair Management de la modélisation. Julien Trufin thanks the financial support of Risk Dynamics under grant New approaches in risk measurement within advanced risk models in actuarial science.

\section{References}

Albrecher, H., Constantinescu, C., \& Loisel, S. (2011). Explicit ruin formulas for models with dependence among risks. Insurance: Mathematics and Economics 48(2), 265-270.

Biard, R., Loisel, S., Macci, C. \& Veraverbeke, N. (2010). Asymptotic behaviour of the finite-time expected time-integrated negative part of some risk processes and optimal reserve allocation. Journal of Mathematical Analysis and Applications 367, 535-549.

Cheridito, P., Delbaen, F., \& Kupper, M. (2006). Coherent and convex monetary risk measures for unbounded cadlag processes. Finance and Stochastics 10, 427-448.

Denuit, M., Dhaene, J., Goovaerts, M.J., \& KaAs, R. (2005). Actuarial Theory for Dependent Risks: Measures, Orders and Models. Wiley, New York.

Dhaene, J., Goovaerts, M.J., \& KaAs, R. (2003). Economic capital allocation derived from risk measures. North American Actuarial Journal 7, 44-59.

Dhaene, J. \& Goovaerts, M.J. (1996). Dependency of risks and stop-loss order. Astin Bulletin 26, 201-212.

Dufresne, F. \& Gerber H.U. (1988). The surpluses immediately before and at ruin, and the amount of claim causing ruin. Insurance: Mathematics and Economics 7, 193-199.

Dos ReIs, A.E. (1993). How long is the surplus belong zero? Insurance: Mathematics and Economics 12, 23-38.

Gerber, H.U. (1988). Mathematical fun with ruin theory. Insurance: Mathematics and Economics 7, 15-23.

Gerber, H.U., Goovaerts, M., \& KaAs, R. (1987). On the probability and severity of ruin. Astin Bulletin 17, 151-163.

Loisel, S. (2005). Differentiation of some functionals of risk processes, and optimal reserve allocation. J. Appl. Prob. 42(2), 379-392. 
MÜLLER, A. (1997). Stop-loss order for portfolios with dependent risks. Insurance: Mathematics and Economics 21, 219-223.

PiCARD, P. (1994). On some measures of the severity of ruin in the classical Poisson model. Insurance: Mathematics and Economics 14, 107-115.

Shaked, M., \& Shanthikumar, J.G. (2007). Stochastic Orders. Springer, New York.

Trufin, J., Denuit, M., \& Albrecher, H. (2011). Properties of a risk measure derived from ruin theory. The Geneva Risk and Insurance Review 36(2), 174-188. 